08

\title{
Влияние интегрированных сопротивлений, созданных под действием ионного облучения, на сверхпроводящие переходы нанопроводников из нитрида ниобия
}

\author{
(C) Б.А. Гурович, ${ }^{1}$ К.Е. Приходько, ${ }^{1,2}$ Б.В. Гончаров, ${ }^{1,}{ }^{\top}$ М.М. Дементьева, ${ }^{1}$ Л.В. Кутузов, ${ }^{1}$ Д.А. Комаров, ${ }^{1}$ \\ А.Г. Домантовский, ${ }^{1}$ В.Л. Столяров, ${ }^{1}$ Е.Д. Ольшанский ${ }^{1}$
}

${ }^{1}$ Национальный исследовательский центр „Курчатовский институт“, 123182 Москва, Россия

${ }^{2}$ Национальный исследовательский ядерный университет (МИФИ), 115409 Москва, Россия

ฯ e-mail: goncharov_bv@nrcki.ru

Поступило в Редакцию 1 апреля 2020 г.

В окончательной редакции 1 апреля 2020 г.

Принято к публикации 1 апреля 2020 г.

Исследовано влияние интегрированных сопротивлений, созданных под действием облучения в нанопроводе, на его сверхпроводящие переходы. Рассмотрены нанопровода из нитрида ниобия шириной $75-20000 \mathrm{~nm}$, изготовленные из пленки $\mathrm{NbN}$ толщиной $5 \mathrm{~nm}$ на подложках из монокристаллического кремния, покрытого слоем термического оксида кремния толщиной $0.3 \mu \mathrm{m}$ и на подложках из сапфира. Описано влияние встроенной резистивной области на величину критического тока перехода из сверхпроводящего состояния в нормальное.

Ключевые слова: тонкие сверхпроводящие пленки $\mathrm{NbN}$, критические токи перехода сверхпроводящих нанопроводов, криоэлектронные устройства, интегрированные криогенные резисторы, ионное облучение.

DOI: $10.21883 /$ JTF.2020.11.49975.106-20

\section{Введение}

Нано- и микроразмерные проводники, изготовленные из тонких пленок $\mathrm{NbN}$, получили широкое распространение в области криоэлектроники. На их основе создан широкий спектр устройств, таких как сверхпроводниковые однофотонные детекторы SNSPD, болометры на „горячих электронах“ $\mathrm{HEB}$, а также элементы логических схем. Создание логических элементов с использованием изменения проводимости отдельных участков нанопроводников было продемонстрированно в работе [1]. В НИЦ „Курчатовский институс“ была продемонстрирована технология, позволяющая локально изменять проводимость при помощи ионного облучения, в том числе создавать области, характеризующиеся металлической проводимостью при температуре жидкого гелия [2,3]. Данная технология позволяет создавать резистивные элементы, интегрированные в сверхпроводящие тоководы, а также позволяет избежать трудностей, связанных с согласованием резистивных элементов со сверхпроводящим тоководом и существенно сократить число необходимых технологических операций для создания каждого элемента [4]. Созданные таким образом сопротивления могут быть использованы в качестве токоограничивающих элементов в составе сверхпроводниковых однофотонных детекторов с разрешением числа фотонов PNR, либо как элементы логических схем. Однако встроенные вышеописанным методом резистивные элементы оказывают существенное влияние на величину критического тока всего нанопроводника, что требует дополнительного изучения.

В настоящей работе изучено влияние встроенной резистивной области на величину критического тока перехода нанопроводника из сверхпроводящего состояния в нормальное.

\section{1. Эксперимент}

В качестве исходного материала, для изготовления нанопроводов, были использованы тонкие пленки $\mathrm{NbN}$, полученные методом катодного распыления. Толщина пленок составила $5 \mathrm{~nm}$. Использовались подложки из монокристаллического кремния, покрытого слоем термического оксида кремния толщиной $0.3 \mu \mathrm{m}$, и подложки из сапфира. Образцы для измерения вольт-амперных характеристик (BAX) представляли собой „микромосты“ длинной $20 \mu \mathrm{m}$ различной ширины от 1 до $20 \mu \mathrm{m}$, а также нанопровода длинной $\sim 10 \mu \mathrm{m}$ и шириной $350 \mathrm{~nm}$ (рис. 1,a). Структуры формировались с использованием методов оптической и электронной литографии, а также реактивного ионного травления [5]. Также на образцах были сформированы макроскопические измерительные металлические контакты $(\mathrm{Ni} / \mathrm{Pt})$ (рис. 1,b). Контакты для измерений были созданы методом катодного распыления.

Для формирования встроенного сопротивления часть нанопровода подвергалась воздействию смешанного ионного облучения (ионами $\mathrm{H}^{+}$и $\mathrm{OH}^{+}$) через защитную 


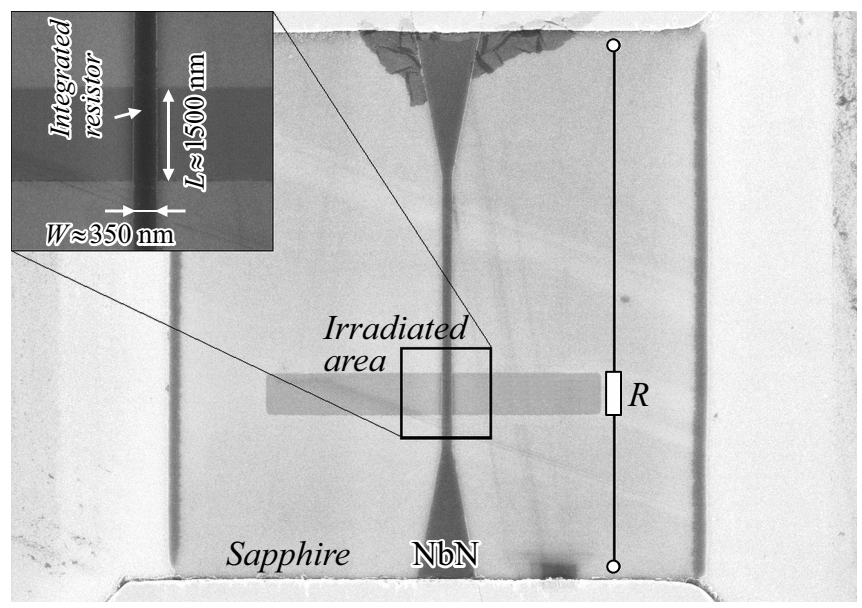

$b$

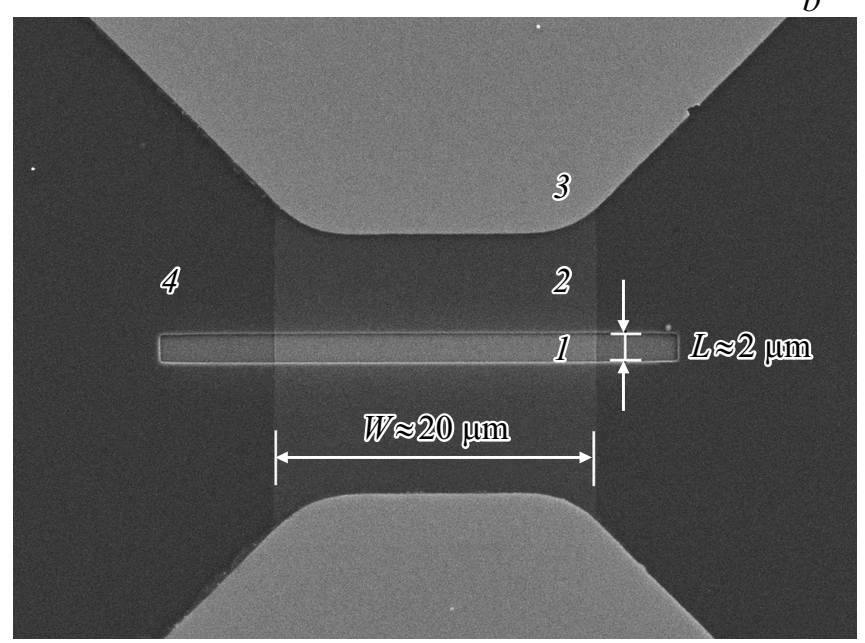

Рис. 1. СЭМ изображения нанопроводника, изготовленного из пленки $\mathrm{NbN}$ (содержащего модифицированную облучением область), шириной $W=350 \mathrm{~nm}(a)$, шириной $W=20 \mu \mathrm{m}(b)$ ( 1 - модифицированная область, 2 - проводник из $\mathrm{NbN}$, 3 - металлические $(\mathrm{Pt})$ измерительные макроконтакты, 4 подложка).

маску, в результате в облученной области формировался участок нормального металла [3]. Доза облучения составляла 2-5 смещений на атом (с.н.а.) (по азоту), состав пучка контролировался путем контроля парциальных давлений газов в вакуумной камере, итоговое сопротивление на квадрат $\left(R_{s q}\right)$, модифицированной области лежало в диапазоне $850-1600 \Omega /$ sq. Все параметры облучения были выбраны исходя из данных, полученных в [4].

Величина встроенного сопротивления, сформированного таким методом, определяется как

$$
R=R_{s q} L / W,
$$

где $R_{s q}-$ сопротивление на квадрат облученной области, $L$ и $W$ - длина и ширина облученной области соответственно.
Измерения проводились в жидком гелии при температуре подложки $4.2 \mathrm{~K}$. Измерение ВАХ осуществлялось 4-контактным методом. Измерительные зонды прижимались к макроскопическим контактам. Контактным сопротивлением можно пренебречь, поскольку площадь измерительных контактов всегда была много больше площади исследуемой структуры. Также это косвенно подтверждается величиной остаточного сопротивления на ВАX исходных (необлученных) образцов, которое составляло во всех случаях $1-4 \Omega$.

\section{2. Результаты и обсуждение}

Основной целью исследования было выявление влияния сопротивлений, встроенных в сверхпроводящий нанопровод, на параметры его перехода из сверхпроводящего состояния в нормальное и обратно. Главным изучаемым параметром являлся критический ток и критическая плотность тока соответствующих переходов.

Для исходного сверхпроводящего нанопроводника, не содержащего встроенного сопротивления, обозначим ток прямого перехода из сверхпроводящего состояния в нормальное $(S \rightarrow N)$ как $I_{1}$, а ток обратного перехода из нормального состояния в сверхпроводящее $(N \rightarrow S)$ как $I_{2}$. Для нанопроводников, изготовленных из тонких пленок, характерна большая разница между значениями токов $I_{1}$ и $I_{2}$, т. е. они проявляют сильный гистерезис по току (рис. 2).

Измерения BAX нанопроводников, содержащих встроенное сопротивление, показали, что прямой переход сверхпроводящего нанопровода, из сверхпроводящего состояния в нормальное $(S \rightarrow N)$ происходит не при токе $I_{1}$, как было до встраивания сопротивления в нанопровод, а существенно раньше - при значениях тока $I_{R}$, примерно соответствующих току возврата исходного, не содержащего встроенного сопротивления, нанопроводника из нормального в сверхпроводящее состояние $I_{2}(N \rightarrow S)$. Данный эффект наблюдался для всех исследованных геометрий проводников (рис. 2).

Измеренные значения критической плотности тока $\left(j_{2}\right)$ перехода исходных нанопроводов, не содержащих интегрированные сопротивления, из нормального состояния в сверхпроводящее, рассчитанные с учетом площади поперечного сечения $S$ :

$$
j_{2}=I_{2} / S,
$$

составили $5.5 \cdot 10^{5} \mathrm{~A} / \mathrm{cm}^{2}$ для проводников с шириной $350 \mathrm{~nm}$ и $1.57 \cdot 10^{6} \mathrm{~A} / \mathrm{cm}^{2}$ для проводников шириной $20 \mu \mathrm{m}$.

На рис. 3 построена зависимость отношения критической плотности тока перехода из сверхпроводящего в нормальное состояние сверхпроводника, содержащего интегрированное сопротивление $\left(j_{r}=I_{R} / S\right)$, к критической плотности тока без сопротивления $j_{2}$. Видно, что отношение $j_{r} / j_{2}$ слабо зависит от номинала встроенного сопротивления $R$. 

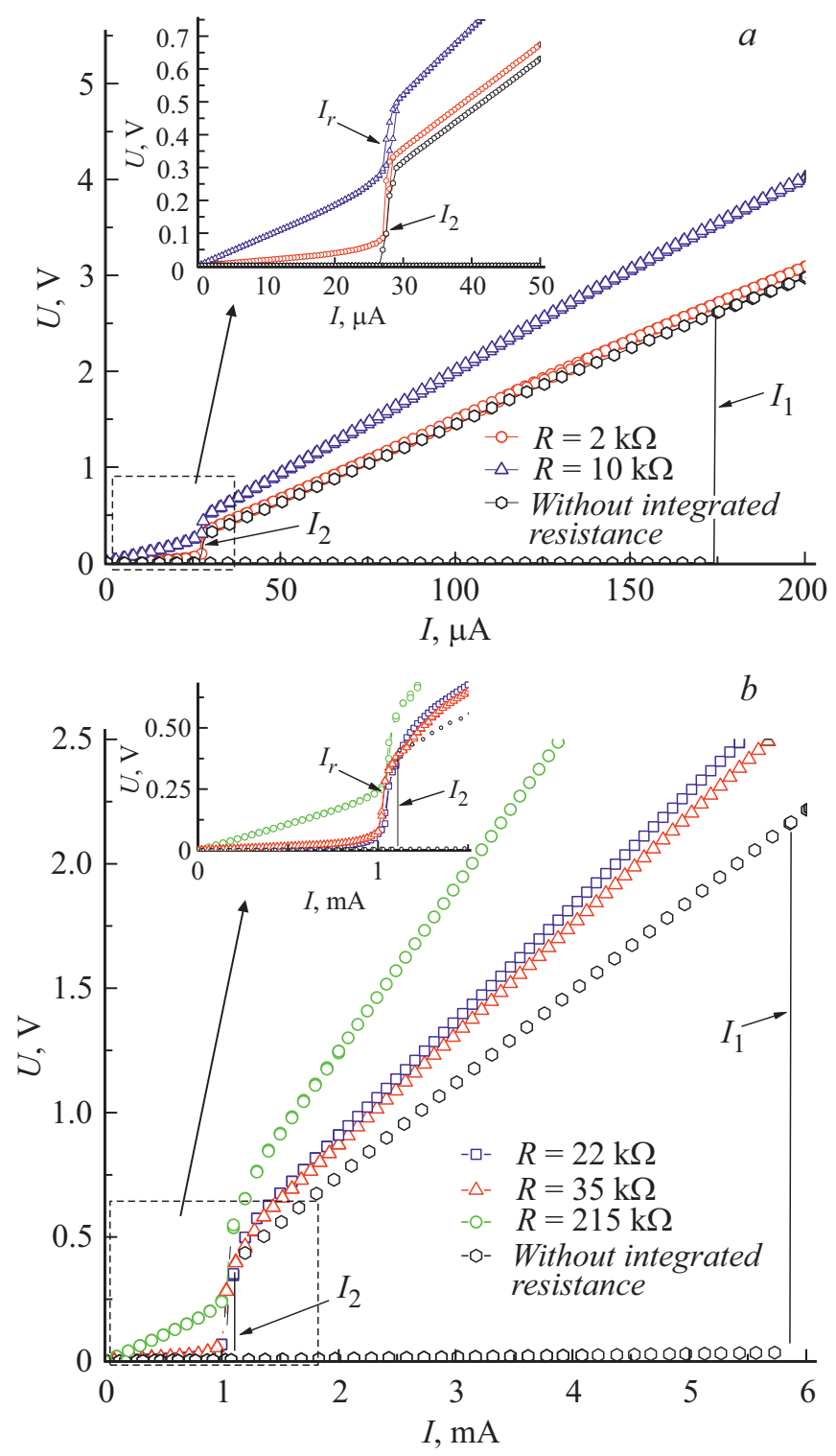

Рис. 2. Измеренные ВАХ нанопроводников с различной величиной интегрированного сопротивления, шириной $W=350 \mathrm{~nm}(a)$, шириной $W=20 \mu \mathrm{m}(b)$.

Природа обратного перехода нанопровода без интегрированного сопротивления из нормального состояния в сверхпроводящее при уменьшении тока через него, по-видимому, состоит в том, что тепловой мощности, выделяемой в нормальном металле, при определенных значениях тока не хватает для поддержания температуры выше критической с учетом теплосъема окружающей среды, что и определяет величину тока обратного перехода нанопровода [6]. При наличии интегрированного в нанопровод сопротивления выделение в нем тепла происходит при любом значении тока. В таком нанопроводе прямой переход из сверхпроводящего состояния в нормальное происходит при значениях тока, близких к величине тока обратного перехода нанопровода, без интегрированного сопротивления, так как заранее со-

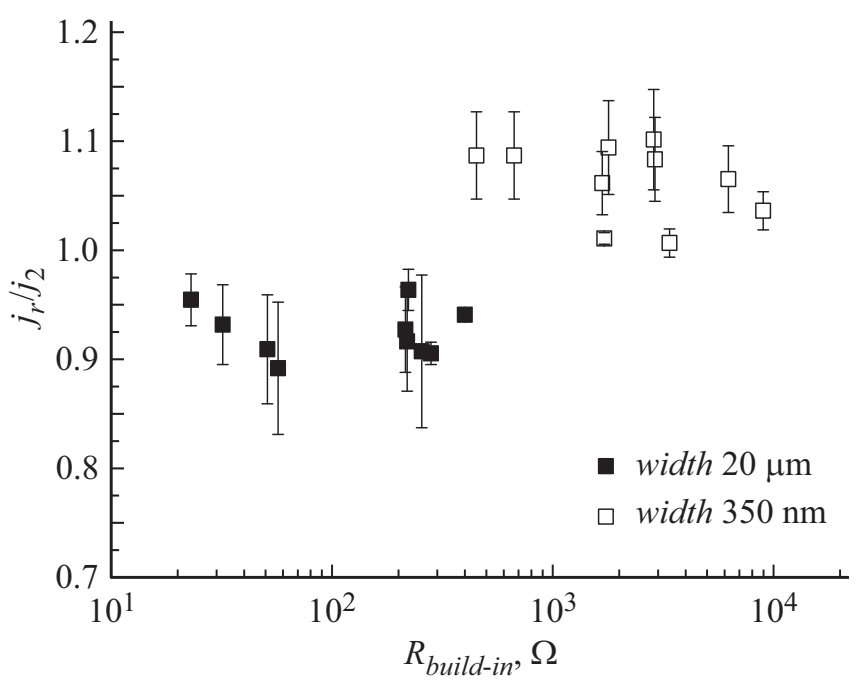

Рис. 3. Зависимость значений критической плотности тока $j_{r}$ (для $S \rightarrow N$ перехода), отнесенной к величине критической плотности тока $j_{2}$ (для $N \rightarrow S$ перехода), для различных $R$ и разной ширины нанопроводника.

зданная область нормального металла является постоянным источником тепла. Отсутствие влияния номинала $R$ на ток $I_{R}$, по-видимому, объясняется тем, что этот переход обусловлен разогревом нанопровода до критической температуры на границе раздела сопротивление/нанопровод, а для заданной ширины нанопровода величины интегрированных $\mathrm{R}$ пропорциональны их длине $L$. При этом площадь поверхности теплоотвода от сопротивления в подложку или во внешнюю среду также $\sim L$. Последнее, по-видимому, является главной причиной отсутствия влияния номинала $R$ на $I_{R}$.

Данный эффект может быть использован при создании криоэлектронных логических схем для управления критическим током и гистерезисом в переключаемых сверхпроводящих элементах.

\section{Финансирование работы}

Работа выполнена при поддержке Национальный исследовательский центр „Курчатовский институт“ (приказ от 25.06.2019 № 1359).

\section{Конфликт интересов}

Авторы заявляют, что у них нет конфликта интересов.

\section{Список литературы}

[1] McCaughan A.N., Berggren K.K. // Nano Lett. 2014. Vol. 14. N 10. P. 5748.

[2] Пат. № 2694799 РФ. Способ уменьшения критического тока перехода наноразмерного сверхпроводника из сверхпроводящего состояния в нормальное / Б.А. Гурович, К.Е. Приходько, А.Г. Домантовский, Е.А. Кулешова, Л.В. Кутузов. заявл. 25.10.2018; опубл. 16.07.2019. Б. 20. 11 с. 
[3] Gurovich B.A., Prikhodko K.E., Tarkhov M.A., Domantovsky A.G., Komarov D.A., Goncharov B.V., Kuleshova E.A. // Micro Nanosystems. 2015. Vol. 8. P. 1-8.

[4] Gurovich B.A., Goncharov B.V., Dementyeva M.M., Prikhodko K.E., Kutuzov L.V., Komarov D.A., Domantovsky A.G. // IOP Conf. Ser. Mater. Sci. Eng. 2019. Vol. 699. P. 012016.

[5] Gurovich B.A., Prikhod'ko K.E., Tarkhov M.A., Kuleshova E.A., Komarov D.A., Stolyarov V.L., Ol'shanskii E.D., Goncharov B.V., Goncharova D.A., Kutuzov L.V., Domantovskii A.G., Lavrukhina Z.V., Dement'eva M.M. // Nanotechnol. Russ. 2015. Vol. 10. N 7-8. P. 530

[6] Tinkham M., Free J.U., Lau C.N., Markovic N. // Phys. Rev. B. 2003. Vol. 68. P. 134515. 